\title{
World Journal of Pediatric Surgery \\ Association of anesthetic and surgical risk factors with outcomes of initial diagnostic biopsies in a current cohort of children with anterior mediastinal masses
}

Huma Faiz Halepota, ${ }^{1,2}$ Josephine S K Tan, ${ }^{3}$ Satish K Reddy, ${ }^{3}$ Phua Hwee Tang, ${ }^{4}$ Lin Yin Ong, ${ }^{1}$ York Tien Lee, ${ }^{1}$ Mei Yoke Chan, ${ }^{5}$ Shui Yen Soh, ${ }^{5}$ Kenneth T E Chang, ${ }^{6}$ Agnes S B Ng, ${ }^{3}$ Amos Hong Pheng Loh (D) ${ }^{1}$

To cite: Halepota HF, Tan JSK, Reddy SK, et al. Association of anesthetic and surgical risk factors with outcomes of initial diagnostic biopsies in a current cohort of children with anterior mediastinal masses. World Jnl Ped Surgery 2021;4:e000303. doi:10.1136/wjps-2021-000303

Received 2 May 2021 Accepted 6 October 2021
Check for updates

C) Author(s) (or their employer(s)) 2021. Re-use permitted under CC BY-NC. No commercial re-use. See rights and permissions. Published by BMJ.

For numbered affiliations see end of article.

Correspondence to Dr Amos Hong Pheng Loh; amos.loh.h.p@singhealth. com.sg

\section{ABSTRACT}

Background Diagnostic biopsies of pediatric anterior mediastinal masses (AMMs) are high-risk procedures in which general anesthesia $(\mathrm{GA})$ is traditionally avoided. However, awareness of historically recognized risk factors and corresponding perioperative management have improved over time and may now no longer strictly preclude the use of GA. Therefore, in this study, we examined the association of anesthetic and surgical risk factors and modalities with resulting procedural and survival outcomes in a current patient cohort.

Methods We retrospectively reviewed charts of 35 children with AMMs who underwent initial diagnostic biopsies between January 2001 and August 2019, and determined tracheal compression and deviation from archival CT scans and procedural and disease outcomes. Results Twenty-three (65\%) patients underwent GA while $12(35 \%)$ received sedation. Among patients with available CT measurements, 13 of $25(52 \%)$ had $>50 \%$ anteroposterior tracheal diameter reduction. Patients with $>50 \%$ anteroposterior tracheal compression received sedation more frequently $(p=0.047)$ and were positioned upright $(p=0.015)$ compared with patients with $\leq 50 \%$ compression, although 4 of 13 and 9 of 12, respectively, still received GA. Intraoperative adverse events (AEs) occurred in four (11.4\%) patients: three received GA, and all were positioned supine or lateral. AEs were not associated with radiographic airway risk factors but were significantly associated with morphine and sevoflurane use $(p<0.001)$ and with thoracoscopic biopsies $(p=0.035)$. There were no on-table mortalities, but four delayed deaths occurred (three related to disease and one from late procedural complications).

Conclusions In a current cohort of pediatric AMM biopsies, patients with $>50 \%$ anteroposterior tracheal compression were more frequently managed with a conservative perioperative management strategy, though not completely excluding GA. The corresponding reduction in frequency of procedural AEs in this traditionally high-risk group suggests that increased awareness of procedural risk factors and appropriate risk-guided perioperative management choices may obviate the procedural mortality historically associated with pediatric AMM biopsies.

\section{Key messages}

What is already known about this subject?

- Historically, general anesthesia (GA) has been strictly avoided for anterior mediastinal mass (AMM) biopsies.

- Evolving awareness of airway risk factors has improved identification of patients suitable for biopsies under GA.

- Radiographic, rather than clinical features, better indicate the actual extent of airway compromise by AMMs in children.

\section{What are the new findings?}

- In a current cohort, most patients with AMM with $>50 \%$ tracheal compression appropriately had biopsies under sedation and in upright position.

- Procedural adverse effects were more frequent among patients who were positioned supine or had thoracoscopic biopsies.

- Ketamine and dexmedetomidine have been used increasingly for sedation in these procedures.

How might it impact on clinical practice in the foreseeable future?

- Historically associated mortality in pediatric AMM may be obviated now by increased awareness of airway risk factors.

- Risk-guided perioperative management choices can permit selective GA use in lower risk patients with AMM.

- Procedural measures and drugs that optimize muscle tone and spontaneous ventilation may improve safety of pediatric AMM biopsies.

\section{INTRODUCTION}

Diagnostic biopsies of anterior mediastinal masses (AMMs) carry significant operative and anesthetic risk. ${ }^{1}$ The mass effect of these tumors can lead to tracheal or bronchial compression and to interruption of venous return or cardiac output, with potentially 
catastrophic consequences. ${ }^{2}$ However, while these biopsies are high-risk procedures, they cannot be avoided for many AMMs because histological and molecular analyses are still required for the prescription of appropriate therapy.

Well-described clinical and radiographic risk features have been highlighted as red flags for potential procedural mortality, especially tracheal compression. ${ }^{3} 4$ Following the initial recognition of these procedural risk factors in the 1970s, general anesthesia (GA) and invasive ventilation had traditionally been strictly avoided. ${ }^{5}$ Yet, conducting these procedures solely under local anesthesia is difficult in children due to their anxiety and inability to cooperate with invasive procedures when awake. ${ }^{6}$ In more recent literature, strategies such as upright posturing, newer anesthetic drugs and better selections of drug combinations have been shown to mitigate these risks. ${ }^{7}$ Thus, with evolving awareness of historical procedural risk factors, accurate estimation of individual patients' risk level and appropriate selection of perioperative approaches may facilitate safe conduct of these biopsies without strictly precluding the use of GA. However, the outcomes of these procedures performed with current day management strategies have not been reviewed in recent literature.

Thus, we aimed to study the association of airway risk factors with choices of anesthetic and surgical approaches and the resulting procedural and survival outcomes, in a current cohort of pediatric patients with AMMs undergoing initial diagnostic biopsies.

\section{MATERIALS AND METHODS}

\section{Patients}

We retrospectively reviewed charts of all patients with AMMs treated at KK Women's and Children's Hospital between January 1, 2001 and August 31, 2019. We included all patients aged 16 years or younger at diagnosis who underwent diagnostic biopsies as the initial surgical procedure in the operating room. Data collected included demographic characteristics, imaging features, tumor location, procedural details, intraoperative complications, histopathological diagnosis, proceduralrelated outcomes (morbidity or mortality), and long-term survival outcome. Adverse events were graded according to Common Terminology Criteria for Adverse Events (CTCAE) V.5.0 criteria.

\section{Radiologic assessment}

AMMs were characterized initially with standard chest radiographs followed by CT of the chest. Digitized CT images were reviewed on Carestream Vue Radiology information system, Picture Archiving, and Communication System (PACS) workstation (V.12.2.1), and measurements were taken digitally.

CT images were reviewed retrospectively by a consultant pediatric radiologist blinded to clinical metadata to determine the extent of tracheal anteroposterior compression, as defined by King $e t a l,{ }^{8}$ and tracheal deviation, a possible risk feature of interest. For the latter, deviation was defined when one edge of the trachea crossed the midline to the contralateral side. ${ }^{9}$ For the former, the smallest anteroposterior tracheal diameter was expressed as a percentage of the maximum normal tracheal diameter, measured at the level of the thoracic inlet at a segment of trachea with no evidence of extrinsic compression by the tumor. This permitted evaluation of tracheal dimensions over the entire intrathoracic segment rather than only a few selected points. ${ }^{1011}$

\section{Procedural measures}

All patients with newly diagnosed AMMs were managed by multidisciplinary teams of oncologists, surgeons, anesthetists, and radiologists. Management plans for all cases were based on consensus recommendations of multidisciplinary tumor boards. Symptomatic patients were monitored in the high dependency or critical care unit as needed. For analysis, GA was defined as any use of inhalational anesthetic agents with placement of an invasive airway.

\section{Statistical methods}

Categorical variables were compared using $\mathrm{X}^{2}$ test, using SPSS V.17.0 (IBM). Fisher's exact test was used in place of $\mathrm{X}^{2}$ test when cells had an expected number of frequencies fewer than 5. For comparisons of more than two groups, post-hoc Bonferroni-adjusted z-tests were performed to determine specific subset pairs that differed significantly at the 0.05 level. Differences were considered significant at $\mathrm{p}<0.05$.

\section{RESULTS}

We identified 35 children with AMMs: 23 (65.7\%) were male and $12(34.3 \%)$ were female; diagnoses included lymphoma $(\mathrm{N}=19)$, neuroblastoma $(\mathrm{N}=5)$, germ cell tumor $(\mathrm{N}=4)$ and other masses $(\mathrm{N}=7)$ that included tuberculosis, Langerhans cell histiocytosis, pleuropulmonary blastoma, aneurysmal bone cyst, congenital pulmonary airway malformation, leukemia and bacterial lymphadenitis. In total, 23 (65\%) underwent GA and 12 $(35 \%)$ had sedation. No procedures were performed only with local anesthesia. Biopsy approach was percutaneous in 19 (54\%), open in $12(34 \%)$, and thoracoscopic in $4(11 \%)$ cases. Digital PACS measurements could be performed on CT scans of 25 patients. Among them, 13 $(52 \%)$ had $>50 \%$ reduction in tracheal anteroposterior diameter and $10(40 \%)$ had tracheal deviation.

Significantly more patients with $>50 \%$ tracheal anteroposterior compression had a diagnosis of lymphoma (11 $(85 \% \%)$ vs $4(33 \%), \mathrm{p}=0.026)$, received sedation $(9(69 \%)$ vs $3(25 \%), \mathrm{p}=0.047)$, and were positioned upright $(6$ $(46 \%)$ vs $0, p=0.015)$ compared with patients with $\leq 50 \%$ compression (table 1). Among them, nine patients $(69 \%)$ were either spontaneously ventilated and/or put in a sitting position (figure 1). These anesthetic measures 


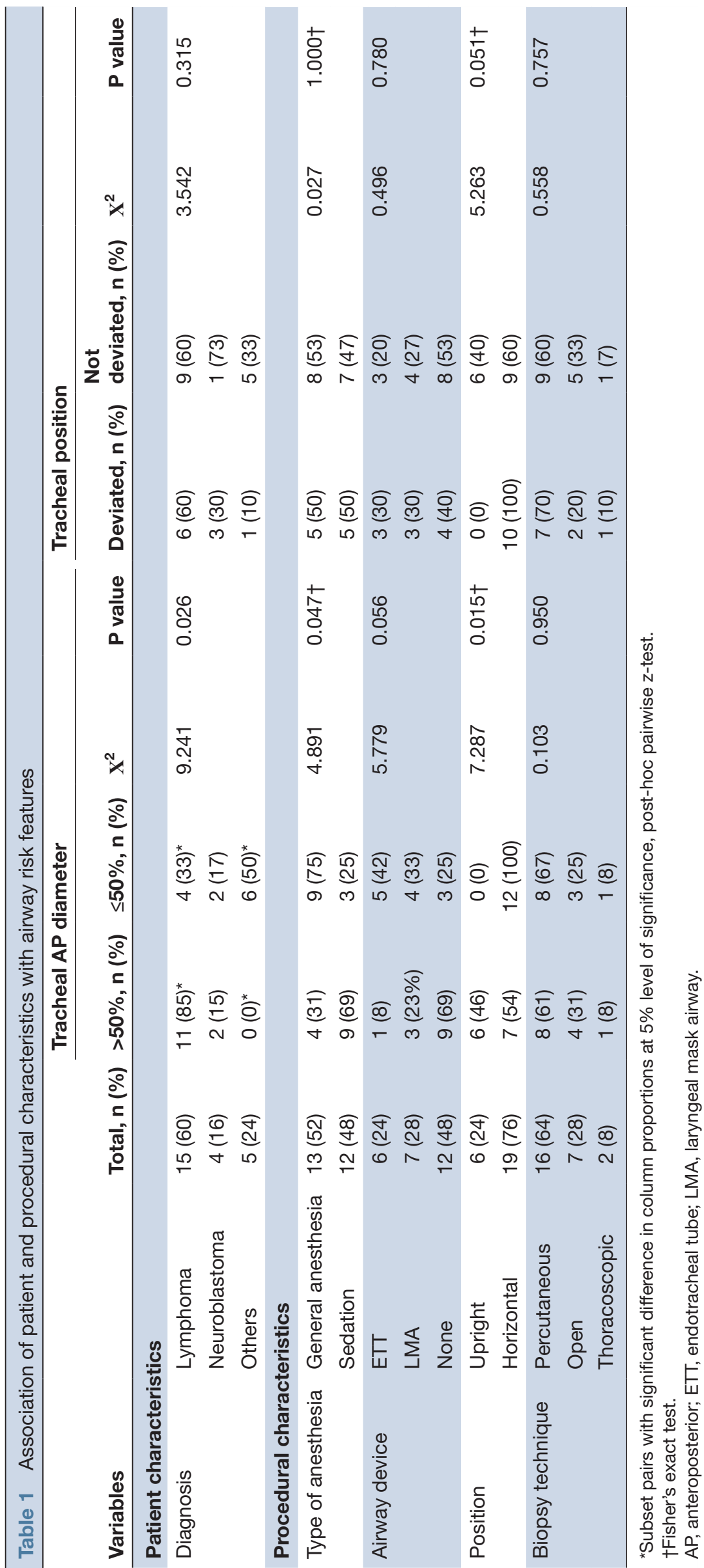


Anesthetic drugs, airway devices, and positions utilized in anterior mediastinal mass biopsies over time

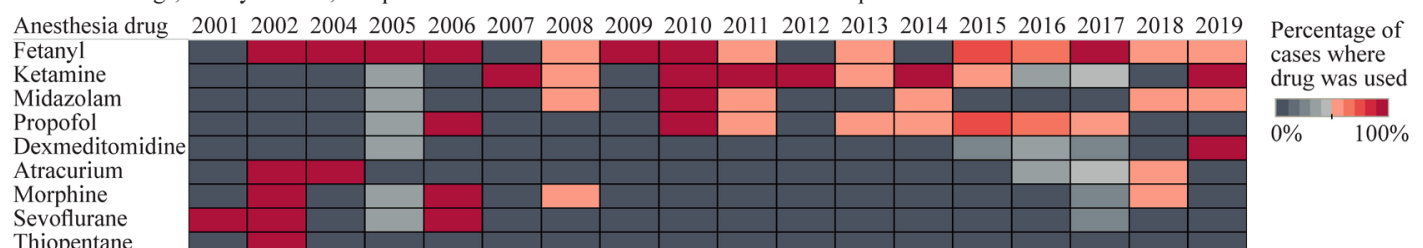

Thiopentane

\section{Airway device} use per case
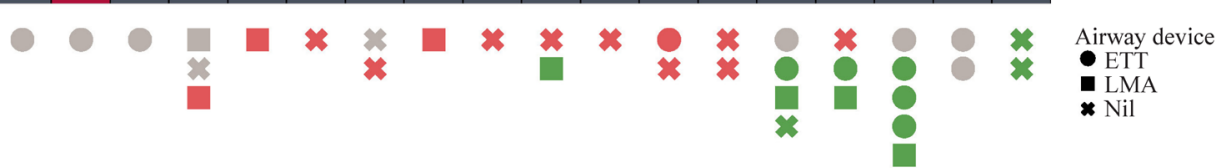

$$
\begin{aligned}
& \text { Position and } \\
& \text { tracheal } \\
& \text { diameter per } \\
& \text { case }
\end{aligned}
$$

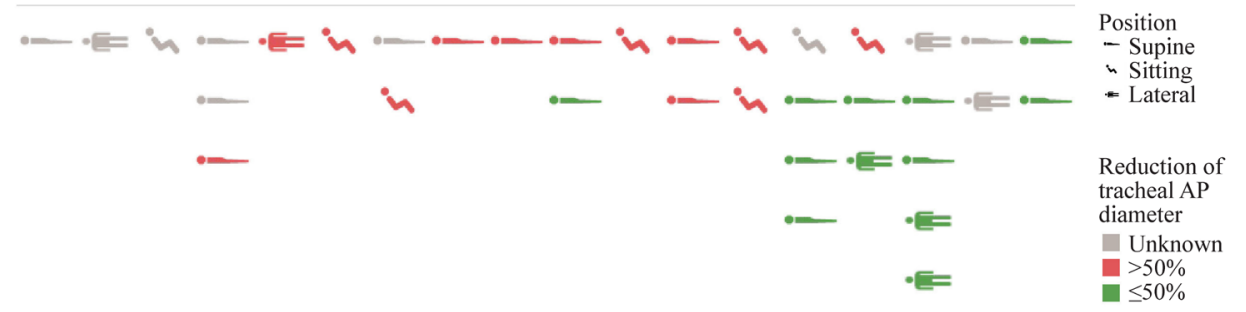

Figure 1 Density plot demonstrating relative use of nine anesthetic drugs per case per year, from 2001 to 2019 (upper panel), and pictographs demonstrating definitive airway devices and positioning used in 35 patients, with the corresponding degree of tracheal anteroposterior (AP) diameter narrowing in each case (color scale) (middle and lower panels). ETT, endotracheal tube; LMA, laryngeal mask airway.

did not differ significantly with presence or absence of tracheal deviation (table 1).

Among patients who underwent biopsies under sedation, supportive measures commonly employed included preprocedural counseling of family and patients, bedside mock rehearsals of anticipated conscious procedures, use of pre-induction topical anesthesia at intended biopsy and intravenous cannulation sites, and intravenous anxiolysis (figure 2).

In total, 4 of $35(11.4 \%)$ patients encountered procedural adverse events: 2 underwent thoracoscopic biopsy; 3 received GA; all 4 were CTCAE grade 1 and 2 events. Notably, these patients were not placed upright for their biopsies, with three of four in the lateral position (table 2). The first patient, a 10-year-old with lymphoma and a history of asthma, developed laryngospasm after induction with intravenous thiopentone but thereafter successfully underwent thoracoscopic biopsy in a right lateral position. The second patient, a 3-year-old with lymphoma, presented with superior vena cava obstruction. Following inhalational induction with sevoflurane, he developed laryngospasm and desaturated, then was deepened with propofol. He then underwent an open biopsy in a supine position. The third patient, a 12-year-old with lymphoma, presented with mediastinal and tracheal deviation. He had transient desaturation during inhalational induction with sevoflurane but therafter could be ventilated uneventfully, and a thoracoscopic biopsy was performed under GA in lateral position. The fourth patient with pleuropulmonary blastoma presented with tracheal deviation and $>50 \%$ tracheal compression. She underwent percutaneous biopsy in lateral position under ketamine sedation but during attempted central line insertion desaturated and was intubated. She failed extubation, later requiring high-frequency oscillatory ventilation and then extracorporeal membrane oxygenation
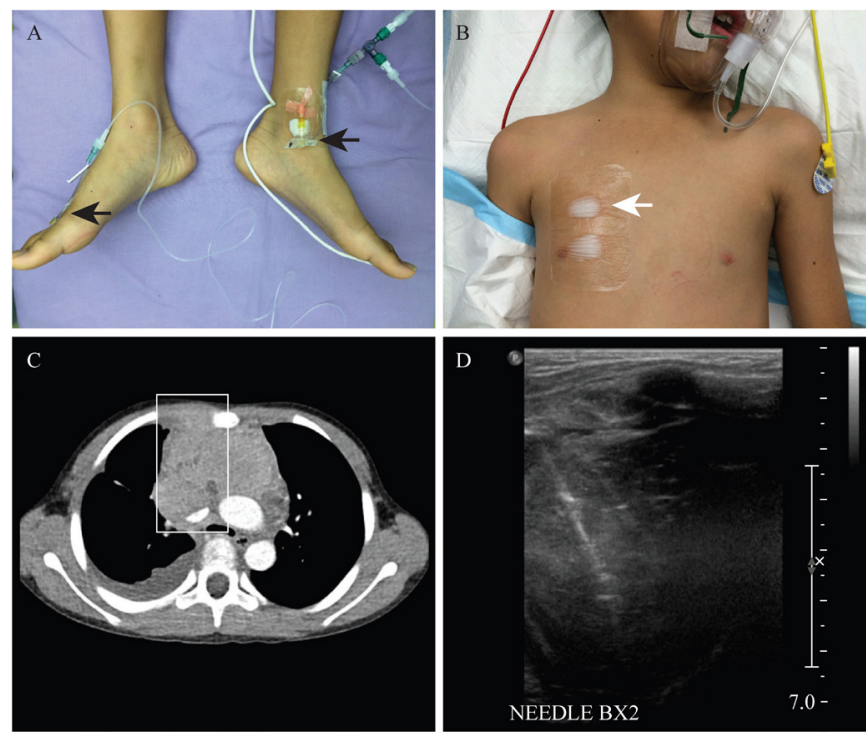

Figure 2 Photographs of multimodal interventions employed on a representative patient with anterior mediastinal mass, including: (A) large-bore venous access in lower limbs (arrows), and (B) maintenance of spontaneous respiration via conscious sedation with preprocedural topical anesthetic applied at potential biopsy sites (arrow); (C,D) representative axial contrast-enhanced CT image of the same patient at the selected biopsy site, and corresponding intraoperative sonographic image of percutaneous core needle biopsy corresponding to an indicated region of interest on CT image. 


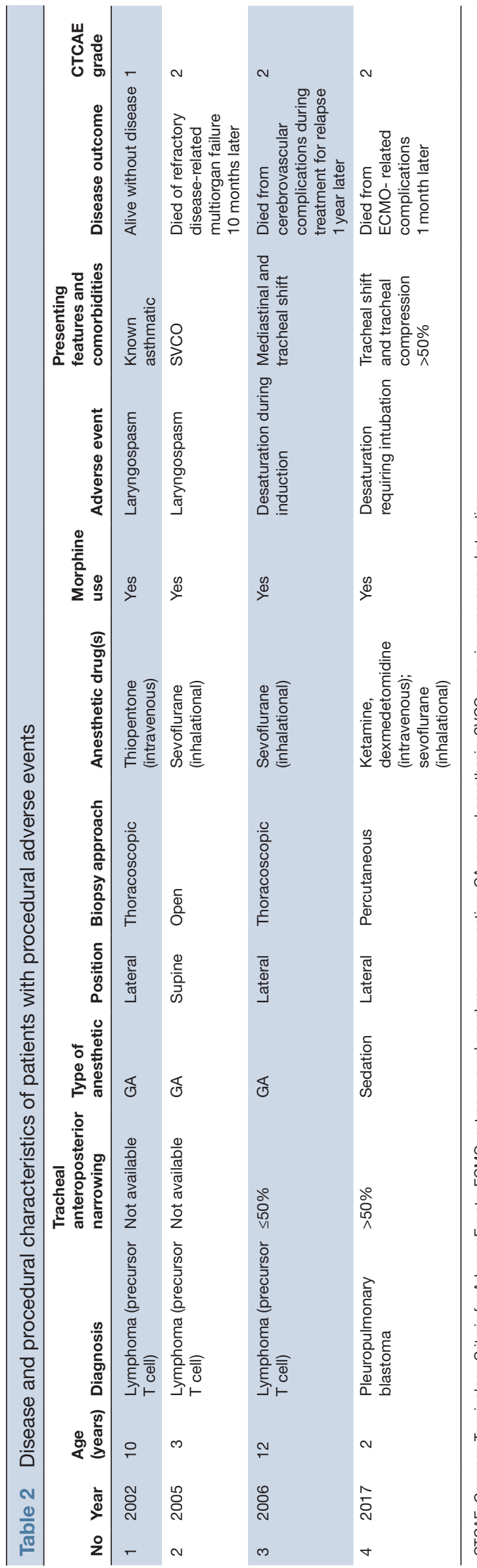

(ECMO). She died 1 month post-biopsy due to ECMOrelated complications.

Overall incidence of procedural-related adverse events did not differ with airway risk factors but was significantly higher in patients who had morphine and sevoflurane $(p<0.001)$ and in those with thoracoscopic biopsies $(\mathrm{p}=0.035) \quad$ (table 3$)$. No direct surgical complications were encountered. Regarding long-term survival, in total, four deaths were encountered: three were delayed and related to disease progression and one (patient 4) was temporally related to the biopsy procedure (table 2). Among the study variables analyzed, only tracheal deviation was significantly associated with long-term survival outcome $(\mathrm{p}=0.017) \quad($ table 3$)$.

\section{DISCUSSION}

In our current experience with initial diagnostic biopsies of pediatric patients with AMM, airway risk factors, such as anteroposterior tracheal compression, were not associated with increased incidence of procedural adverse events. Increased adverse events were instead observed with thoracoscopic biopsies and with the use of sevoflurane and morphine. Our findings suggest that with greater awareness of airway risk factors, appropriate patient selection and risk-avoidance practices may effectively obviate the procedural mortality historically associated with these procedures.

Predictive risk factors for mortality from AMM biopsies have evolved during recent decades. The association of anesthetic risk with CT-detected tracheal cross-sectional area was first identified in the 1980s, with avoidance of GA recommended for all children with $<50 \%$ $66 \%$ normal tracheal area. ${ }^{3}{ }^{10}$ In the 1990 s, recognition of significant risk factors for periprocedural mortality evolved to include $<50 \%$ predicted peak expiratory flow rate (PEFR) and reduced tracheal cross-sectional area, together termed as 'Shamberger's box'. ${ }^{11} 12$ In patients with mediastinal lymphoma, the presence of clinical symptoms was additionally associated with increased anesthetic complications. ${ }^{8}$ This study found that anesthetic and surgical management choices of managing teams reflected appropriate risk-based considerations, and procedural morbidities that were historically associated with patients with these risk factors correspondingly decreased. More recent literature is consistent with our experience and may reflect more current trends in anesthetic and surgical practice, particularly that clinical symptoms and PEFR correlate poorly with the actual extent of anatomical airway obstruction and eventual complications. ${ }^{13}$ Interestingly, we observed that long-term survival was correlated with tracheal deviation, which could be a radiographic risk feature that might indicate more aggressive tumor behavior or greater tumor burden.

Our practice experience supports the benefits of multidisciplinary management of these complex cases. ${ }^{14-16}$ Preoperative assessments and formulation of multidisciplinary plans have been shown to effectively guide 


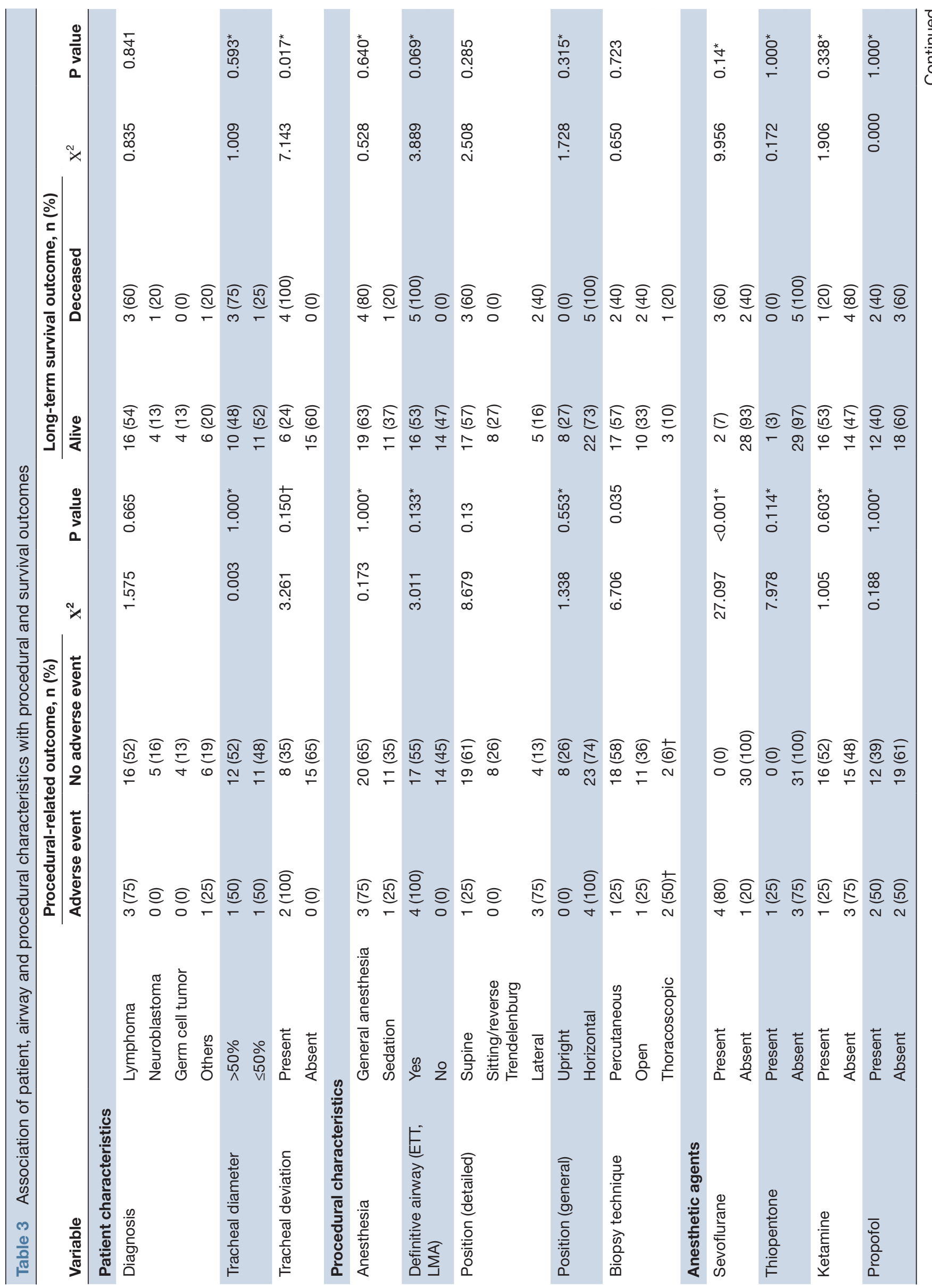




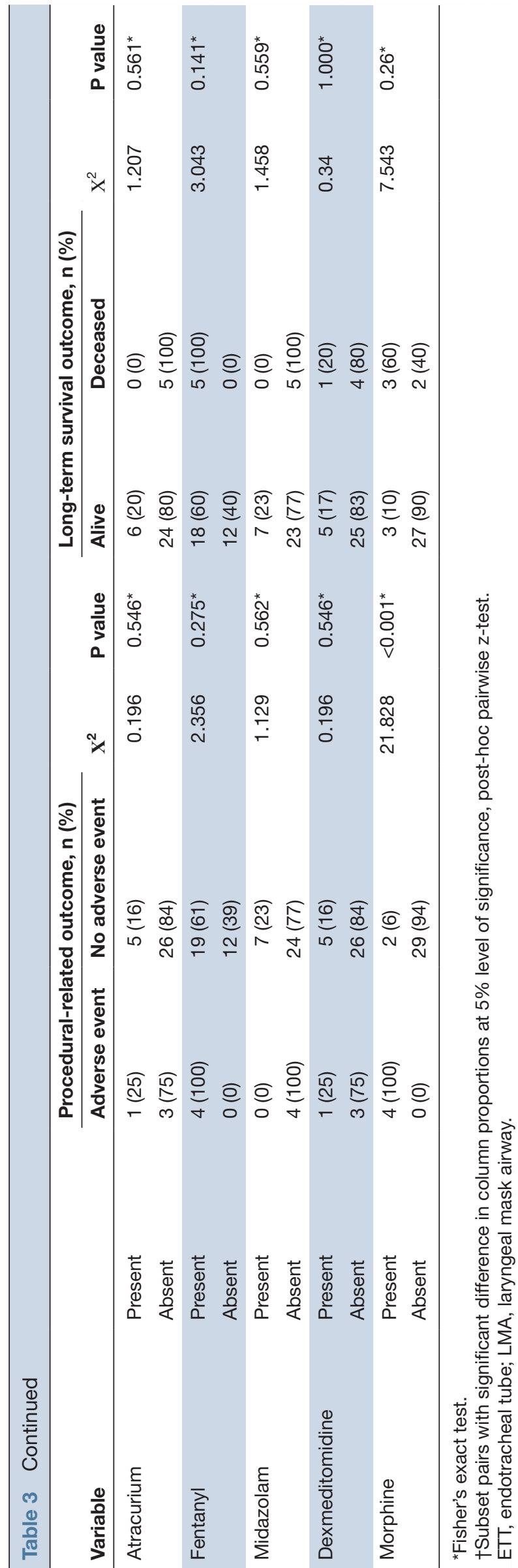

management in such patients and to prevent overall morbidity and mortality. ${ }^{17}$ If anesthetic or surgical expertise is insufficient to provide the required support for an AMM biopsy, safer alternative sites, such as bone marrow, pleural fluid and extrathoracic lymph nodes, can be considered instead. ${ }^{18}$ However, they are associated with false negative or inaccurate histological results, ${ }^{19-22}$ with diagnostic yields as low as 32\% reported in bone marrow aspirates and $86 \%$ from lymph node biopsies in Hodgkin's disease. ${ }^{23}$ In lymphoblastic lymphoma, even less invasive means such as pleural fluid analysis can be used to further mitigate risks. ${ }^{24}$

Aggressive surgical interventions, such as the use of cardiopulmonary bypass or attempts at upfront resection, have very limited utility and need to be employed with caution. Given the success of the more conservative initial diagnostic approaches that we demonstrated, these more radical maneuvers may hold even less relevance in current practice and if so, only in a few selected scenarios. ${ }^{25}$ However, empirical upfront treatment also can lead to dramatic tumor shrinkage, which may make AMMs inaccessible for biopsy or potentially alter the histomorphology. ${ }^{18}$ In pediatric patients with mediastinal lymphoma, pre-biopsy steroids have been associated with compromised pathological diagnosis in up to $22 \%$ of $\operatorname{cases}^{26}$ but should be considered particularly in patients presenting with critical life-threatening airway obstruction. ${ }^{27}$ Pre-biopsy radiation can also distort cellular morphology and compromise accurate histological diagnosis and should be considered with caution. $^{28}$

Historically, GA has been strictly discouraged in patients with AMMs because of the risk of precipitating complete airway obstruction due to tracheal collapse, ${ }^{29} 30$ with up to $7 \%-20 \%$ encountering complications, ranging from transient hypotension to complete cardiorespiratory collapse. ${ }^{31}$ In our study, at-risk patients with $>50 \%$ reduction in tracheal anteroposterior diameter had significantly greater use of sedation over GA, with corresponding reduction of procedural morbidities. This supports recommendations to maintain muscle tone and spontaneous ventilation in such patients. ${ }^{32}$ Also, in our study, procedural adverse events were significantly higher in those who positioned supine. This supports the use of upright posturing to decrease anteroposterior pressure on the trachea, thereby avoiding critical airway collapse. In the event of inadvertent intraprocedural deterioration, possible contingencies can include increasing fractional inspired oxygen, repositioning, continuous positive airway pressure or intermittent positive pressure ventilation, rigid bronchoscopy or reducing anesthetic depth. $^{33} 34$

During the study period, we observed changing trends in the use of sedative agents in our practice. There was an increase in the use of ketamine from 2007 onwards, possibly due to increased experience with its use in patients with AMM and to increased awareness of its advantages, namely maintenance of intercostal muscle 
function and chest wall tone, which prevent respiratory collapse. ${ }^{35}$ Also, we noted an increase in the use of dexmedetomidine, which also caused minimal respiratory depression. ${ }^{36}$ However, to date, there has been little published experience with the use of dexmedetomidine in pediatric AMMs. ${ }^{36-39}$ Increasingly, we have been combining dexmedetomidine with ketamine, for their complementary analgesic and sympatholytic effects.

This study was limited as a single-center experience and by the small patient numbers, which limited the extent of statistical analysis, particularly precluding multivariable analysis. The wide range of diagnoses included in the study may limit the interpretability of long-term survival data, though it would be expected to have less direct impact on immediate procedural outcomes compared with the anatomical configuration of the tumor and resulting airway compromise. While guided by current evidence, choice of specific procedural measures was not enforced among individual anesthetists and surgeons. The duration of the study period could have contributed to temporal bias and to selection bias due to inclusion of only digitized CT studies. Using risk-based algorithms and guidelines for multidisciplinary teams, future research could study the impact of protocol-based treatment plans in these high-risk procedures. Additionally, future studies could evaluate recently described novel predictive risk factors, including standardized tumor volume and mediastinal mass ratio, which is the CT-measured proportion of maximal widths of mass over mediastinum. ${ }^{40}$

In conclusion, through a more selective approach to using GA, traditionally high-risk patients with $>50 \%$ anteroposterior tracheal compression could safely undergo initial diagnostic biopsies of AMMs. Increased appreciation of radiographic risk factors for airway-related procedural morbidity and risk-adapted perioperative management may help to reduce the adverse outcomes historically associated with pediatric AMM biopsies.

\section{Author affiliations \\ ${ }^{1}$ Department of Paediatric Surgery, KK Women's and Children's Hospital, Singapore ${ }^{2}$ Department of Paediatric General Surgery, The Indus Hospital, Karachi, Sindh, Pakistan \\ ${ }^{3}$ Department of Paediatric Anesthesia, KK Women's and Children's Hospital, Singapore \\ ${ }^{4}$ Department of Diagnostic and Interventional Imaging, KK Women's and Children's Hospital, Singapore \\ ${ }^{5}$ Haematology/Oncology Service, Department of Paediatric Subspecialties, KK Women's and Children's Hospital, Singapore \\ ${ }^{6}$ Department of Pathology and Laboratory Medicine, KK Women's and Children's Hospital, Singapore}

Acknowledgements The authors thank Ms Tan Sheng Hui, VIVA-KKH Paediatric Brain and Solid Tumour Programme, and Ms Candy Choo, Department of Pediatric Surgery, KK Women's and Children's Hospital.

Contributors HFH contributed to data curation and writing - original draft. JSKT contributed to data curation and writing - review and editing. SKR and ASBN contributed to formal analysis and writing - review and editing. PHT performed data curation, formal analysis and writing - review and editing. LYO, MYC and KTEC contributed to writing - review and editing. YTL contributed to data curation. SYS contributed to resources and writing - review and editing. AHPL was the guarantor, contributed to supervision, data curation, formal analysis, writing - review and editing and resources.

Funding This work was supported by the VIVA Foundation for Children with Cancer, Singapore (award number: NA, award title: VIVA-KKH Paediatric Brain and Solid Tumour Programme) (AHPL), and Children's Cancer Foundation, Singapore (award number: NA, award title: Singapore Childhood Cancer Registry) (SYS).

Competing interests None declared.

Patient consent for publication Patient consent for publication was obtained.

Ethics approval This study was approved by SingHealth Central Institutional Review Board (CIRB protocol 2019/2234) and granted waiver of informed consent.

Provenance and peer review Not commissioned; externally peer reviewed.

Data availability statement Data are available upon reasonable request. The data that support the findings of this study are available on request from the corresponding author. The data are not publicly available due to information that could compromise the privacy of research participants.

Open access This is an open access article distributed in accordance with the Creative Commons Attribution Non Commercial (CC BY-NC 4.0) license, which permits others to distribute, remix, adapt, build upon this work non-commercially, and license their derivative works on different terms, provided the original work is properly cited, appropriate credit is given, any changes made indicated, and the use is non-commercial. See: http://creativecommons.org/licenses/by-nc/4.0/.

ORCID iD

Amos Hong Pheng Loh http://orcid.org/0000-0001-8333-1486

\section{REFERENCES}

1 Slinger P, Karsli C. Management of the patient with a large anterior mediastinal mass: recurring myths. Curr Opin Anaesthesiol 2007;20:1-3.

2 Keon TP. Death on induction of anesthesia for cervical node biopsy. Anesthesiology 1981;55:469-71.

3 Shamberger RS, Holzman RS, Griscom NT, et al. Ct quantitation of tracheal cross-sectional area as a guide to the surgical and anesthetic management of children with anterior mediastinal masses. J Pediatr Surg 1991;26:138-42.

4 Mandell GA, Lantieri R, Goodman LR. Tracheobronchial compression in Hodgkin lymphoma in children. AJR Am J Roentgenol 1982;139:1167-70.

5 Amaha K, Okutsu Y, Nakamura Y. Major airway obstruction by mediastinal tumour. A case report. Br J Anaesth 1973;45:1082-4.

6 Sibert KS, Biondi JW, Hirsch NP. Spontaneous respiration during thoracotomy in a patient with a mediastinal mass. Anesth Analg 1987;66:904-7.

7 Tanaka T, Amano H, Tanaka Y, et al. Safe diagnostic management of malignant mediastinal tumors in the presence of respiratory distress: a 10-year experience. BMC Pediatr 2020;20:1-6.

8 King DR, Patrick LE, Ginn-Pease ME, et al. Pulmonary function is compromised in children with mediastinal lymphoma. J Pediatr Surg 1997;32:294-300.

9 Ishii H, Kinoshita Y, Kushima H, et al. The upward shift of hilar structures and tracheal deviation in pleuroparenchymal fibroelastosis. Multidiscip Respir Med 2019;14:10.

10 Azizkhan RG, Dudgeon DL, Buck JR, et al. Life-Threatening airway obstruction as a complication to the management of mediastinal masses in children. J Pediatr Surg 1985;20:816-22.

11 Shamberger RC. Preanesthetic evaluation of children with anterior mediastinal masses. Semin Pediatr Surg 1999;8:61-8.

12 Shamberger RC, Holzman RS, Griscom NT, et al. Prospective evaluation by computed tomography and pulmonary function tests of children with mediastinal masses. Surgery 1995;118:468-71.

13 Garey CL, Laituri CA, Valusek PA, et al. Management of anterior mediastinal masses in children. Eur J Pediatr Surg 2011;21:310-3.

14 Mushtaq N, Alam MM, Aslam S, et al. Malignant mediastinal mass in children: a single institutional experience from a developing country. J Pak Med Assoc 2014;64:386-9.

15 Unceta-Barrenechea Orúe B, Garrán Sabando B, Reguera Fernández MA, et al. [Mediastinal tumors in children: apropos of 2 cases]. Rev Esp Anestesiol Reanim 1990;37:168-71.

16 Gun F, Erginel B, Unüvar A, et al. Mediastinal masses in children: experience with 120 cases. Pediatr Hematol Oncol 2012;29:141-7.

17 Freud E, Ben-Ari J, Schonfeld T, et al. Mediastinal tumors in children: a single institution experience. Clin Pediatr 2002;41:219-23. 
18 Perger L, Lee EY, Shamberger RC. Management of children and adolescents with a critical airway due to compression by an anterior mediastinal mass. J Pediatr Surg 2008;43:1990-7.

19 Acker SN, Linton J, Tan GM, et al. A multidisciplinary approach to the management of anterior mediastinal masses in children. $J$ Pediatr Surg 2015;50:875-8.

20 McCarten KM, Metzger ML, Drachtman RA, et al. Significance of pleural effusion at diagnosis in pediatric Hodgkin lymphoma: a report from children's Oncology Group protocol AHOD0031. Pediatr Radiol 2018;48:1736-44.

21 Sethuraman C, Simmerson M, Vora AJ, et al. Flowcytometric immunophenotyping in the diagnosis of pediatric lymphoma: how reliable is it and how can we optimize its use? J Pediatr Hematol Oncol 2010;32:298-303.

22 McLean TW, Farber RS, Lewis ZT, et al. Diagnosis of Burkitt lymphoma in pediatric patients by Thoracentesis. Pediatr Blood Cancer 2007;49:90-2.

23 Simpson I, Campbell PE. Mediastinal masses in childhood: a review from a paediatric pathologist's point of view. Prog Pediatr Surg 1991;27:92-126.

24 Chaignaud BE, Bonsack TA, Kozakewich HP, et al. Pleural effusions in lymphoblastic lymphoma: a diagnostic alternative. J Pediatr Surg 1998;33:1355-7.

25 Tempe DK, Arya R, Dubey S, et al. Mediastinal mass resection: femorofemoral cardiopulmonary bypass before induction of anesthesia in the management of airway obstruction. $J$ Cardiothorac Vasc Anesth 2001;15:233-6.

26 Borenstein SH, Gerstle T, Malkin D, et al. The effects of prebiopsy corticosteroid treatment on the diagnosis of mediastinal lymphoma. J Pediatr Surg 2000;35:973-6.

27 Sakaguchi G, Tachibana K, Takeuchi M, et al. [Decision to avoid general anesthesia on 3 cases with anterior mediastinal masses]. Masui 2011;60:609-14.
28 Ferrari LR, Bedford RF. General anesthesia prior to treatment of anterior mediastinal masses in pediatric cancer patients. Anesthesiology 1990;72:991-5.

29 Gothard JWW. Mediastinal Mass - Anesthetic Considerations. Anesthesiol Clin 2008;26:305-14.

30 Ricketts RR. Clinical management of anterior mediastinal tumors in children. Semin Pediatr Surg 2001;10:161-8.

31 Pearson JK, Tan GM. Pediatric anterior mediastinal mass. Semin Cardiothorac Vasc Anesth 2015;19:248-54

32 Malik R, Mullassery D, Kleine-Brueggeney M, et al. Anterior mediastinal masses - A multidisciplinary pathway for safe diagnostic procedures. J Pediatr Surg 2019;54:251-4

33 McLeod M, Dobbie M. Anterior mediastinal masses in children. BJA Educ 2019;19:21-6.

34 Reddy CSK, Phang DLK, Ng ASB, et al. A simplified approach for anaesthetic management of diagnostic procedures in children with anterior mediastinal mass. Singapore Med J 2020;61:308-11.

35 Drummond GB. Comparison of sedation with midazolam and ketamine: effects on airway muscle activity. $\mathrm{Br} J$ Anaesth 1996;76:663-7.

36 Hasan MS, Ling KU, Chan L. Children with anterior mediastinal mass. Eur J Anaesthesiol 2011;28:813-5.

37 Mahmoud M, Tyler T, Sadhasivam S. Dexmedetomidine and ketamine for large anterior mediastinal mass biopsy. Paediatr Anaesth 2008;18:1011-3.

38 Corridore M, Phillips A, Rabe AJ, et al. Dexmedetomidine-ketamine sedation in a child with a mediastinal mass. World J Pediatr Congenit Heart Surg 2012;3:142-6.

39 Carollo DS, Pestieau S, Bosco R. Dexmedetomidine for anterior mediastinal mass computed tomography-guided biopsy: a case series. Ochsner J 2013;13:541.

40 Kawaguchi Y, Saito T, Mitsunaga T, et al. Prediction of respiratory collapse among pediatric patients with mediastinal tumors during induction of general anesthesia. J Pediatr Surg 2018;53:1365-8. 